\title{
NGHIÊN CỨU ĐỀ XUẤT KHUNG QUY CHUẨN KỸ THUẬT DỮ LIỆ SẢN PHẨM ẢNH VIỄN THÁM
}

\author{
TS. NGUYẼ̃N XUÂN LÂM, ThS. NGUYẼN NGỌC QUANG \\ KS. NGUYẼN TH! NGOCC QUỲNH
}

Cục Viễn thám Quốc gia

\section{Tóm tắt:}

Trên thế giới hiện có 3 tổ chức quốc tế chính về tiêu chuẩn hóa bao gồm Tổ chức quốc tế về chuẩn hóa (ISO), tiểu ban kỹ thuật 211 (TC 211); Ủy ban dữ liệu địa lý liên bang của Hoa Kỳ (FGDC) và Hiệp hội GIS mở (OGC) đã nghiên cứu, xây dựng và ban hành được một số chuẩn liên quan đến dữ liệu viễn thám. Ngoài ra, cơ quan Hàng không vũ trụ Hoa Kỳ (NASA) cũng có những đóng góp không nhỏ trong việc ban hành các tiêu chuẩn này. Hiện tại, cũng chưa có nhiều cơ quan và tổ chức ở Việt Nam bàn cụ thể về vấn đề xây dựng chuẩn dữ liệu viễn thám quốc gia, mới đây Bộ Tài nguyên và Môi trường tuy đã ban hành Quy chuẩn kỹ thuật quốc gia về chuẩn thông tin địa lý cơ sở (QCVN 42: 2012/BTNMT) nhưng bộ Quy chuẩn này cũng chưa đề cập cụ thể cho chuẩn dữ liệu viễn thám. Sau khi nghiên cứu các chuẩn trên thế giới cùng với các văn bản quy phạm pháp luật của Việt Nam có liên quan đến dữ liệu viễn thám nhóm tác giả đã đề xuất được danh mục sáu quy chuẩn kỹ thuật có liên quan đến dữ liệu viễn thám. Bài báo này sẽ tập trung giới thiệu một bộ khung quan trọng và có ý nghĩa thực tiễn cao là khung Quy chuẩn dữ liệu sản phẩm ảnh.

\section{1. Đặt vấn đề}

Trong thời đại công nghệ thông tin hiện nay, dữ liệu không gian địa lý, thông tin địa lý, các ứng dụng dạng thông tin là những tài nguyên quan trọng của xã hội thông tin. Một chức năng quan trọng của các hệ thống và các ứng dụng thông tin địa lý là chia sẻ dữ liệu và giúp người dùng khai thác nguồn tài nguyên này. Các hệ thống cần phải tương thích với nhau để có thể chia sẻ được tài nguyên thông tin. Chìa khóa cho sự thành công của việc cấp phối dữ liệu thông tin địa lý có khả năng tương thích với các hệ thống địa không gian là các tiêu chuẩn (Liping $\mathrm{Di}$, 2003). Viễn thám ngày nay đã trở thành một trong những nguồn dữ liệu thông tin địa lý quan trọng. Hiện tại, Cơ quan Hàng không vũ trụ Hoa Kỳ (NASA) và 3 tổ chức quốc tế chính về tiêu chuẩn hóa bao gồm Ủy ban dữ liệu địa lý liên bang của Hoa Kỳ (FGDC), Hiệp hội GIS mở (OGC) và Tổ chức quốc tế về chuẩn hóa ISO, tiểu ban kỹ thuật 211 (TC 211) đã xây dựng và ban hành các tiêu chuẩn liên quan đến loại dữ liệu viễn thám này. Các chuẩn tiêu biểu và có ảnh hưởng lớn đối với dữ liệu viễn thám có thể kể đến nhu: ISO 19121, ISO 19129 và ISO 19130 của ISO TC211, Chuẩn dữ liệu dải quét và chuẩn dữ liệu ảnh trực giao của FGDC.

Ở Việt Nam, hiện đã có quy định của Nhà nước dành cho loại dữ liệu này cụ thể như: Quyết định số 81 năm 2010 của Thủ tướng Chính phủ quy định về thu nhận, lưu trữ, xử lý, khai thác và sử dụng dữ liệu viễn thám quốc gia. Việc đề cập đến khái niệm "dữ liệu viễn thám quốc gia" đã bước đầu hình thành xây dựng chuẩn cho dữ liệu viễn thám và thống nhất quy định về thu nhận, lưu trữ, xử lý, khai thác và sử dụng nguồn tài nguyên này. Cũng chưa có nhiều cơ quan và tổ chức ở Việt Nam bàn cụ thể về vấn đề xây dựng chuẩn dữ liệu viễn thám quốc gia, mới 
đây Bộ Tài nguyên và Môi trường tuy đã ban hành Quy chuẩn kỹ thuật quốc gia về chuẩn thông tin địa lý cơ sở (QCVN 42: 2012/BTNMT) nhưng bộ Quy chuẩn này cũng chưa đề cập cụ thể cho chuẩn dữ liệu viễn thám. Hiện chưa có một nghiên cứu chính thức nào về xây dựng chuẩn cho dữ liệu viễn thám ở Việt Nam trong khi nguồn tài nguyên này ngày càng được ứng dụng sâu rộng ở nhiều lĩnh vực. Bên cạnh đó, loại dữ liệu viễn thám cũng rất đa dạng và quan trọng hơn cả là số lượng ảnh viễn thám ngày càng trở nên khổng lồ, chỉ tính riêng số lượng dữ liệu ảnh viễn thám SPOT và ENVISAT ở Cục Viễn thám quốc gia, Bộ Tài nguyên và Môi trường đã lên đến hàng nghìn cảnh ảnh (chụp lãnh thổ và biển đảo Việt Nam). Con số này sẽ còn tăng lên nhiều trong một thời gian ngắn nữa vì Trạm thu ảnh viễn thám (Cục Viễn thám quốc gia) đã bắt đầu thu ảnh từ vệ tinh viễn thám VNREDSat-1 đầu tiên của Việt Nam. Vì vậy, việc nghiên cứu xây dựng chuẩn cho dữ liệu viễn thám là cần thiết để phục vụ cho việc quản lý và khai thác sử dụng hiệu quả nguồn tài nguyên này.

\section{Nghiên cứu đề xuất khung quy chuẩn dữ liệu sản phẩm ảnh}

\subsection{Cơ sở khoa học đề xuất khung quy chuẩn dữ liệu sản phẩm ảnh}

Trước khi đi vào việc đề xuất khung quy chuẩn cho dữ liệu sản phẩm ảnh cần phải hiểu khái niệm về dữ liệu sản phẩm ảnh. Theo Quyết định số 81 năm 2010 của Thủ tướng Chính phủ, dữ liệu sản phẩm ảnh được hiểu là dữ liệu ảnh viễn thám đã được xử lý phổ và nắn chỉnh hình học.

Việc xây dựng khung cho dữ liệu sản phẩm ảnh sẽ được tham khảo từ các chuẩn ISO 19121 (Chuẩn dữ liệu dạng ảnh và dạng lưới), ISO 19129 (Chuẩn khung dữ liệu dạng ảnh và dạng lưới có thông tin địa lý), ISO 19130 (Chuẩn mô hình bộ cảm và dữ liệu cho dữ liệu dạng ảnh và dạng lưới) do ISO TC 211 ban hành và Standard for Digital Orthoimagery (Chuẩn cho dữ liệu ảnh trực giao), Standard for Remote Sensing Swath Data (Chuẩn cho dư liệu viễn thám dải quét) do FGDC ban hành.

Nội dung chính của các bộ chuẩn này được miêu tả dưới đây.

\section{Chuẩn ISO 19121}

Bộ chuẩn này đưa ra các đặc tính kỹ thuật của dữ liệu dạng ảnh và dạng lưới. Trong đó, tập trung chính vào định dạng dữ liệu raster và dữ liệu ma trận để hướng tới mục tiêu cung cấp cho người sử dụng định dạng dữ liệu raster chuẩn bởi vì hiện tại mỗi loại vệ tinh đều đưa ra định dạng chuẩn riêng cho mình dựa vào đặc tính kỹ thuật của bộ cảm mà nó sử dụng.

Các mục chính của bộ khung cho chuẩn này bao gồm:

Duyệt lại các chuẩn hiện có

+ Chuẩn về trao đổi dữ liệu không gian dạng raster

+ Chuẩn HDF của NASA

+ Chuẩn cho các định dạng: GIF (The Graphic Interchange Format), PNG (Portable Network Graphics), TIFF (Tag Image File Format) và GEOTIFF.

Thành phần của dữ liệu dạng ảnh và dữ liệu dạng lưới

+ Yếu tố ảnh của các phần tử ảnh

+ Siêu dữ liệu

+ Thông tin không gian

+ Đóng gói và mã hóa dũ liệu.

Tương tác với các chuẩn khác

Chuẩn ISO 19129

Chuẩn này đưa ra định nghĩa cho khung dữ liệu dạng ảnh và dạng lưới có thông tin địa lý. Trong đó đưa ra mô hình cho loại dữ liệu ảnh có thể được biểu diễn dưới dạng 
dữ liệu địa lý. Những mô hình này được trình bày theo ngôn ngữ kiểu UML (Unified Modeling Language) cho các ứng dụng dạng lược đồ.

Các mục chính của bộ khung cho chuẩn này bao gồm:

- Đặc tính chung của mô hình ứng dụng cho dữ liệu dạng ảnh và dạng lưới

+ Đặc tính địa lý

+ Mối liên hệ giữa các đặc tính bổ sung

- Khung

+ Cấu trúc khung

+ Mức mã hóa

+ Miêu tả dữ liệu dạng lưới, dạng khung

+ Bảng tra (LUT) cho mối liên quan giữa các đặc trưng

- Thông tin không gian cho dữ liệu dạng ảnh dạng lưới

- Cấu trúc dữ liệu dạng ảnh dạng lưới có thông tin địa lý

+ Cấu trúc IGCD và siêu dữ liệu

+ Khung cấu trúc lớp dữ liệu

- Mẫu

+ Lược đồ ứng dụng cho dữ liệu dạng ảnh và dạng lưới

+ Lưới cho dữ liệu địa lý

+ Lớp TIN

+ Phân biệt dữ liệu địa lý dạng điểm

+ Phân biệt dữ liệu địa lý dạng bề mặt lưới

\section{Chuẩn ISO 19130}

Chuẩn này mô tả mô hình đầu thu với các thuộc tính vật lý và hình học của phương pháp đo ảnh, viễn thám hoặc các đầu thu khác tạo ra dữ liệu viễn thám. Chuẩn này cũng định nghĩa mô hình dữ liệu khái niệm bao gồm loại đầu thu, các yêu cầu dữ liệu tối thiểu và mối quan hệ giữa các thành phần dữ liệu thô (raw data) được tính toán từ đầu thu và được tích hợp hệ tọa độ của đầu thu để có thể định vị và phân tích được dữ liệu.

Các mục chính của bộ khung cho chuẩn này bao gồm:

- Dữ liệu bộ cảm

+ Mô hình dũ liệu bộ cảm

+ Tổ chức dữ liệu

+ Thành phần mô hình dữ liệu

- Thông tin vị trí địa lý

+ Cấu trúc dữ liệu cho thông tin vị trí địa lý

+ Mô hình bộ cảm

+ Đa mô hình

- Phân loại bộ cảm

+ Mảng quét tuyến tính

+ Bộ cảm Pushbroom

+ Khung camera

+ Quét trên giấy và phim

+ Bộ cảm Radar

+ Bộ cảm Lidar

- Cấu tạo bộ cảm

+ Mô hình bộ cảm

+ Siêu dữ liệu cho bộ cảm

+ Thông số bộ cảm

+ Đặc tính bộ cảm quang học

+ Vị trí và định hướng

- Phân loại dữ liệu raster

- Dữ liệu Radar

Chuẩn FGDC - Standard for Remote Sensing Swath Data

Chuẩn này định nghĩa khái niệm dải quét, các thành phần cơ bản của một dải 
quét, mối quan hệ giữa các thành phần đó. Chuẩn Swath chủ yếu dựa trên khái niệm swath của hệ thống thông tin quan sát trái đất EOSDIS của NASA. Chuẩn này xác định mô hình dữ liệu chuẩn cho dữ liệu swath nhưng không chuẩn hóa việc mã hóa dữ liệu swath.

Các mục chính của bộ khung cho chuẩn này bao gồm:

- Khái niệm dải quét

- Thành phần của dải quét

- Định nghĩa dải quét

+ Dữ liệu bộ cảm

- Loại dữ liệu

- Cấu trúc dữ liệu

- Đơn vị cho dữ liệu

+ Thông tin vị trí

- Thời gian

- Tọa độ địa lý

- Dữ liệu độ cao của vệ tinh

+ Mối quan hệ giữa vị trí địa lý và dữ liệu bộ cảm

Chuẩn FGDC - Standard for Digital

\section{Orthoimagery}

Tiêu chuẩn này đưa ra định nghĩa về ảnh trực giao trong khuôn khổ khung dữ liệu không gian số của FGDC. Mục đích chính là đưa ra một loại chuẩn chung cho sản phẩm ảnh trực giao để đảm bảo cho việc sử dụng dữ liệu rộng rãi, tăng cường chức năng chia sẻ dữ liệu và giảm thiểu việc sản xuất dữ liệu một cách không cần thiết.

Các mục chính của bộ khung cho chuẩn này bao gồm:

- Mô tả dữ liệu

- Định nghĩa dữ liệu ảnh trực giao

- Định dạng truyền dữ liệu
- Nguồn dữ liệu

+ Thời gian/Mùa

+ Ảnh hàng không

+ Ảnh quét từ ảnh hàng không

+ Ảnh viễn thám quang học

+ Dữ liệu độ cao

+ Dữ liệu khống chế

+ Dữ liệu Calibration

- Tham chiếu không gian

- Độ phân giải

+ Độ phân giải không gian

+ Độ phân giải phổ

- Độ chính xác

- Chất lượng dữ liệu

+ Hiệu chỉnh hình học

+ Hiệu chỉnh phổ

- Độ phủ mây

- Ghép ảnh

- Siêu dữ liệu

2.2. Đề xuất khung quy chuẩn dữ liệu sản phẩm ảnh

Qua nghiên cứu và phân tích chỉ có bộ khung cho chuẩn dữ liệu trực giao của FGDC là phù hợp hơn cả cho việc xây dựng khung cho dữ liệu sản phẩm ảnh. Do vậy, việc đề xuất khung cho dữ liệu sản phẩm ảnh sẽ chủ yếu dựa vào bộ khung cho chuẩn dữ liệu trực giao. Dưới đây là nội dung chính của bộ khung quy chuẩn dữ liệu sản phẩm ảnh được đề xuất và ví dụ áp dụng thực tế ở Việt Nam. (Xem bảng 1)

\section{Kết luận và kiến nghị}

Việc nghiên cứu và xây dựng Quy chuẩn kỹ thuật cho dữ liệu viễn thám ở Việt Nam là tương đối mới nên khi thực hiện còn gặp nhiều khó khăn. Tuy nhiên nghiên cứu này 
Bảng 1

\begin{tabular}{|c|c|}
\hline Nội dung chính của khung & Ví dụ áp dụng thực tế \\
\hline $\begin{array}{l}\text { Đặt tên cho dữ liệu sản phẩm } \\
\text { ảnh }\end{array}$ & $\begin{array}{l}\text { Dữ liệu sản phẩm ảnh viễn thám có thể được đặt tên như sau: } \\
\text { V_H_L_NTN_GPG_M } \\
\text { Trong đó: V: tên vệ̂ tinh, H: số hiệu cảnh ảnh, L: loại ảnh (toàn } \\
\text { sắc; đa phổ), NTN: ngày tháng năm chụp ảnh, GPG: giờ phút } \\
\text { giây chụp ảnh, M: mức xử lý ảnh. }\end{array}$ \\
\hline $\begin{array}{l}\text { Thành phần dữ liệu sản phẩm } \\
\text { ảnh }\end{array}$ & $\begin{array}{l}\text { Dũ liệu sản phẩm ảnh viễn thám bao gồm dữ liệu ảnh, ảnh xem } \\
\text { nhanh và thông tin mô tả. Trong đó, ảnh xem nhanh là ảnh có } \\
\text { dung lượng nhỏ ở định dạng jpg cho phép hiển thị nhanh hình } \\
\text { ảnh của dữ liệu ảnh để thuận tiện cho việc tra cứu. }\end{array}$ \\
\hline $\begin{array}{l}\text { Định dạng của dũ liệu sản phẩm } \\
\text { ảnh }\end{array}$ & $\begin{array}{l}\text { Định dạng chuẩn cho dữ liệu sản phẩm ảnh viễn thám phải ở } \\
\text { dạng GEOTIFF; BSQ; BIL hoặc BIP. }\end{array}$ \\
\hline $\begin{array}{l}\text { Mức xử lý cho dũ liệl } \\
\text { ảnh }\end{array}$ & 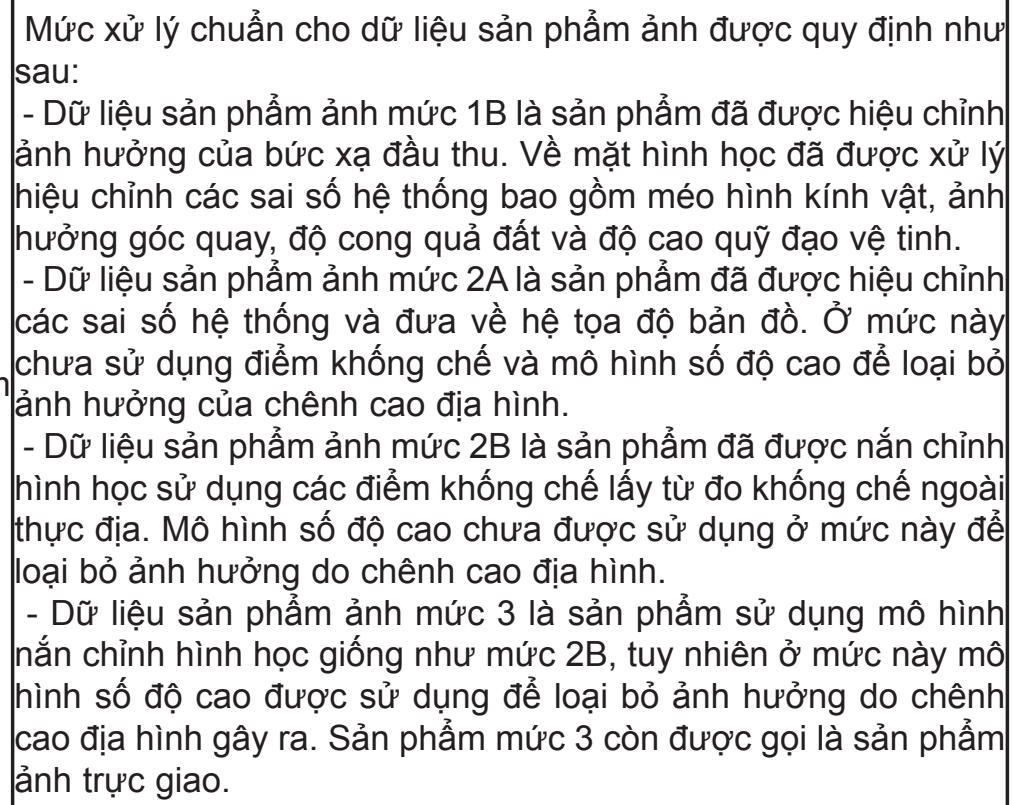 \\
\hline Dũ̃ liệu bổ trợ & $\begin{array}{l}\text { Dũ liệu bổ trợ được sử dụng để tạo ra dữ liệu sản phẩm ảnh. } \\
\text { Dữ liệu này được ghi trong tệp tin siêu dữ liệu viễn thám đi kèm } \\
\text { với dữ liệu ảnh thô. Các dữ liệu cần thiết để tạo ra dữ liệu sản } \\
\text { phẩm ảnh viễn thám bao gồm: } \\
\text { - Dữ liệu ảnh thô } \\
\text { - Thông tin định chuẩn của bộ cảm, thông số kỹ thuật, thông tin } \\
\text { tham chiếu hình học } \\
\text { - Điểm khống chế mặt đất } \\
\text { - Dữ liệu số độ cao }\end{array}$ \\
\hline
\end{tabular}




\begin{tabular}{|c|c|}
\hline Nội dung chính của khung & Ví dụ áp dụng thực tế \\
\hline Chất lượng dữ liệu sản phẩm ảnh & $\begin{array}{l}\text { Dữ liều sản phẩm ảnh phải đảm bảo chất lượng về mặt hình } \\
\text { học và phổ theo quy định kỹ thuật của Cục Viễn thám quốc gia. } \\
\text { Dữ liệu này phải được đưa vào cơ sở dữ liệu viễn thám quốc } \\
\text { gia }\end{array}$ \\
\hline $\begin{array}{l}\text { Độ phù mây của dữ liệu sản phẩm } \\
\text { ảnh }\end{array}$ & $\begin{array}{l}\text { Dữ liệu sản phẩm ảnh viễn thám } \\
10 \% \text { tính trên đơn vị cảnh ảnh (hoặc }\end{array}$ \\
\hline $\begin{array}{l}\text { Siêu dữ liệu cho sản phẩm ảnh: } \\
\text { - Nguồn gốc ảnh } \\
\text { - Thời gian } \\
\text { - Thông tin quỹ đạo } \\
\text { - Loại ảnh } \\
\text { - Độ phân giải } \\
\text { - Số hàng, cột của dữ liệu sản } \\
\text { phẩm ảnh } \\
\text { - Cơ quan, tổ chức, cá nhân xử lý } \\
\text { - Ngày, giờ xử lý } \\
\text { - Mức xử lý } \\
\text { - Định dạng ảnh } \\
\text { - Hệ tọa độ tham chiếu } \\
\text { - Độ chính xác hình học } \\
\text { - Thông tin đô̂ phủ mây } \\
\text { - Cơ quan kiểm tra chất lượng sản } \\
\text { phẩm } \\
\text { - Mục đích sử dụng sản phẩm ảnh }\end{array}$ & $\begin{array}{l}\text { Thông tin của siêu dữ liệu sản phẩm ảnh phải được mô tả } \\
\text { trong tệp tin dạng (.txt) đi kèm với dữ liệu sản phẩm ảnh. } \\
\text { Thông tin mô tả phải có các nội dung như sau: } \\
\text { - Nguồn gốc của ảnh: ảnh được thu từ trạm thu ảnh viễn thám, } \\
\text { mua của nước ngoài... } \\
\text { - Ngày, giờ chụp ảnh } \\
\text { - Số quỹ đạo } \\
\text { - Loại ảnh: ảnh toàn sắc, đa phổ... } \\
\text { - Độ̉ phân giải } \\
\text { - Tồng số hàng, tổng số cột của dữ liệu ảnh } \\
\text { - Cơ quan, tổ chức, cá nhân xử lý } \\
\text { - Ngày, giờ xử lý } \\
\text { - Mức xử lý } \\
\text { - Định dạng ảnh } \\
\text { - Hệ tọa độ tham chiếu } \\
\text { - Độ chính xác hình học } \\
\text { - Thông tin đô̂ phủ mây } \\
\text { - Cơ quan kiểm tra chất lượng sản phẩm ảnh } \\
\text { - Mục đích sử dụng sản phẩm ảnh }\end{array}$ \\
\hline
\end{tabular}

cũng bước đầu tiếp cận và đề xuất được một bộ khung quy chuẩn tương đối hoàn chỉnh liên quan đến dữ liệu viễn thám và có ý nghĩa thực tiễn cao là khung quy chuẩn kỹ thuật dữ liệu sản phẩm ảnh.

Để thuận lợi cho việc xây dựng các bộ quy chuẩn kỹ thuật liên quan đến dữ liệu viễn thám, Cục Viễn thám quốc gia cần ban hành trước các quy định kỹ thuật liên quan đến dữ liệu viễn thám như: Quy định về tên gọi và các thuật ngữ, Quy định về xử lý dữ liệu viễn thám, Quy định về độ chính xác sản phẩm ảnh viễn thám,... Các quy chuẩn kỹ thuật này cần phải được đưa vào xây dựng sớm trong bối cảnh dữ liệu viễn thám ngày càng được sử dụng rộng rãi để thống nhất việc quản lý, khai thác và sử dụng có hiệu quả nguồn tài nguyên quý giá này. $\bigcirc$

\section{Tài liệu tham khảo}

[1]. Luật Tiêu chuẩn và Quy chuẩn kỹ thuật của Quốc hội nước Cộng hòa xã hội chủ nghĩa Việt Nam số 68/2006/QH11đã được thông qua ngày 29 tháng 6 năm 2006 tại kỳ họp thứ 9 , Khóa XI.

[2]. Quy chuẩn kỹ thuật quốc gia về chuẩn thông tin địa lý cơ sở (QCVN 42: 2012/BTNMT).

[3]. Quyết định số 81 ngày 13 tháng 12 năm 2010 của Thủ tướng Chính phủ quy định về thu nhận, lưu trư, xử lý, khai thác và sử dụng dữ liệu viễn thám quốc gia.

[4]. Ad, M., \& Working, H. (1998). 
Content Standard for Digital Geospatial Metadata.

[5]. Di, L., \& Kobler, B. (2000). NASA STANDARDS FOR EARTH REMOTE SENSING DATA, XXXIII(Figure 1), 147-155.

[6]. Di, L. (2003). The development of remote-sensing related standards at FGDC, OGC, and ISO TC 211. IGARSS 2003. 2003 IEEE International Geoscience and Remote Sensing Symposium. Proceedings (IEEE Cat. No.03CH37477), 1(C), 643-647. doi:10.1109/IGARSS.2003.1293868

[7]. Geographic, F., \& Committee, D. (1999a). Content Standards for Digital Orthoimagery, (February).

[8]. Group, S. W., Geographic, F., \& Committee, D. (2002a). Content Standard for Digital Geospatial Metadata: Extensions for Remote Sensing Metadata.
[9]. Group, S. W., Geographic, F., \& Committee, D. (2002b). Content Standard for Digital Geospatial Metadata : Extensions for Remote Sensing Metadata.

[10]. Iso, S. G., \& Information, G. (2009). STANDARDS GUIDE ISO / TC 211 GEOGRAPHIC INFORMATION / GEOMATICS.

[11]. Kennedy, E. M., Sundquist, D. O. N., Chairman, V., Hollings, E. F., Carolina, S., Dingell, J. D., ... Doan, H. T. E. D. (1993). Data Format Standards for Civilian Remote Sensing Satellites May 1993 Office of Technology Assessment Congressional Board of the 103d Congress, (May).

[12]. Maitra, J. (2004). Standards of ISO Technical Committee 211 Geographic information / Geomatics Presented by Julie Binder Maitra, GSDI-7 Tutorial, Bangalore, India. O

\section{Summary}

\section{Research on building technical regulations for remotely sensed data}

Dr. Nguyen Xuan Lam, MSc. Nguyen Ngoc Quang, Eng. Nguyen Thi Ngoc Quynh

\section{Vietnam Remote Sensing Center}

Nowadays, there has been 3 official orgnizations of standardization, including International Orgnization for Standardization (ISO), Technical Committee 211 (TC211), Federal Geographic Data Committee (FGDC), Open Geospatial Consortium (OGC) and National Aeronautics and Space Administration (NASA) researched and resulted in some standards relating to remote sensing data. Building technical regulations for remote sensing data have not been deployed in Vietnam so far, recently Ministry of Natural Resources and Environment has approved National technical regulation on standard of basic geographic information but it has not mentioned in remotely sensed data yet. This paper will focus on building one of the most important standard frameworks that related to remote sensing data and suited for application in Vietnam. $O$

Ngày nhận bài: 20/12/2013. 